\title{
Evaluation de la reproductibilité des réponses hydraulique et épuratoire de deux noues pilotes
}

\author{
Alexandre Fardel ${ }^{1,2}$, Pierre-Emmanuel Peyneau ${ }^{1}$, Béatrice Béchet $^{1}$, Abdelkader Lakel $^{2}$ \\ et Fabrice Rodriguez ${ }^{1, *}$ \\ ${ }^{1}$ IFSTTAR, GERS, LEE, route de Bouaye CS4, 44344 Bouguenais, France \\ ${ }^{2}$ CSTB, 11 rue Henri Picherit, 44300 Nantes, France
}

Reçu le 22 mars 2019 / Accepté le 7 mai 2019

\begin{abstract}
Résumé - Deux noues végétalisées ont été mises en œuvre au sein d'un pilote au CSTB (Nantes), et ont été équipées d'un simulateur de ruissellement et d'une instrumentation dédiée afin d'en étudier leurs réponses hydraulique et épuratoire. La fonctionnalité de la chaîne de mesure et la reproductibilité des réponses des noues ont été évaluées sur un même évènement de ruissellement (pluie d'environ $11 \mathrm{~mm}$ ), simulé à 6 reprises entre novembre 2017 et avril 2018. Le dispositif instrumental s'est avéré apte à restituer intégralement la réponse hydraulique des deux noues, et sa capacité à suivre finement les flux de polluants en entrée et en sortie de noue a été validée. Les valeurs brutes des ratios flux d'eau sortant/injecté (S/E) et flux d'eau infiltré/ruisselé (I) sont faiblement dispersées (coefficients de variation $(\mathrm{cv})<5 \%$ ). Les abattements en masse et en concentration des deux polluants analysés dans cette étude, le zinc $(\mathrm{Zn})$ et le cuivre $(\mathrm{Cu})$, sont compris dans les limites de reproductibilité calculées. En revanche, le niveau de contamination élevé de $\mathrm{Zn}$ dans les eaux injectées pourrait expliquer la plus faible dispersion de son abattement $(\mathrm{cv}<30 \%)$ par rapport à $\mathrm{Cu}$ $(-185 \% \leq \mathrm{cv} \leq 176 \%)$. Enfin, les incertitudes liées à la mesure et aux méthodes analytiques ont été identifiées comme des sources potentielles de variations des réponses. Ces résultats seraient à approfondir pour des conditions initiales plus contrastées (pluies extrêmes, sol sec ou proche de la saturation).
\end{abstract}

Mots clés : noues / eaux pluviales / réponse hydraulique / performances épuratoires / instrumentation

Abstract - Evaluation of hydraulic and pollutant filtration response replicability of two pilot swales. Swales are popular stormwater source-control infrastructures put in place to mitigate the environmental impacts of urban or road runoff. Over the past 30 years, swale design has evolved with more emphasis on swale outflow quality, but still has a strong empirical basis. Therefore, there is a need to gain insight into the design aspects that could impinge on the treatment efficiency of a swale. To address these knowledge gaps, two pilot swales were specifically designed and built at the CSTB Nantes. Differences between the pilot swales derived from their soil materials in order to assess and compare the performances of a standard swale and a filtering swale. For this purpose, the pilot swales were equipped with a runoff simulator and various measuring devices (i.e. flowmeters and soil moisture sensors). Given the importance to generate high-quality data, the present paper details this measurement chain and examines its operation by simulating the same runoff event six times between November 2017 and April 2018. The reproducibility of the swale responses is discussed in light of the test procedure and the estimated measurement uncertainties. Overall, results emphasize the high reproducibility of the swale hydraulic responses and demonstrate that pollutant fluxes can be accurately monitored at the inlet and the outlet of each swale. Regarding hydraulic indicators, slight dispersion (variation coefficient cv $<5 \%$ ) of the ratios between outgoing and incoming water $(\mathrm{S} / \mathrm{E})$ and between infiltrated and runoff water (I) are likely due to non-similar initial conditions (e.g., natural rainfall preceding the runoff event). Regarding performance indicators of the stormwater treatment, the mass and concentration reduction efficiencies for zinc $(\mathrm{Zn})$ and copper $(\mathrm{Cu})$ are within the estimated replicability boundaries. Nevertheless, the high contamination level of $\mathrm{Zn}$ in incoming stormwater could explain the lower dispersion of its removal (cv $<30 \%$ ) with respect to the dispersion of $\mathrm{Cu}$ removal $(-185 \% \leq \mathrm{cv} \leq 176 \%)$. Overall, the estimated uncertainties of both the monitoring devices and the analytical methods were identified as potential explanations for the observed variations.

Keywords: swales / stormwater / hydraulic response / pollutant removal performance / monitoring

\footnotetext{
*Correspondance : fabrice.rodriguez@ifsttar.fr
} 


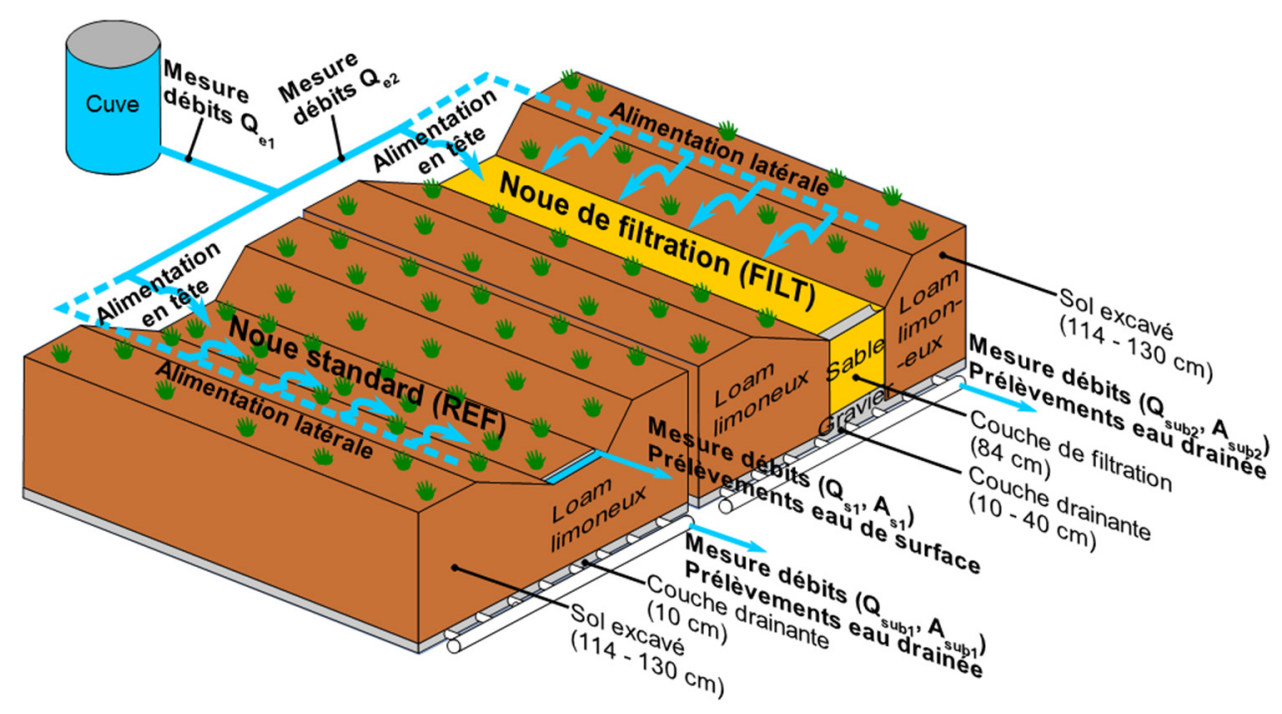

Fig. 1. Schéma du pilote aménagé au CSTB. Les débits mesurés par les débitmètres électromagnétiques (DEM) et les Augets présentent respectivement les préfixes $\mathrm{Q}$ et $\mathrm{A}$.

\section{Introduction}

L'aménagement de techniques alternatives (TA) de gestion des eaux pluviales connaît une croissance rapide depuis les années 1990 pour réduire les risques d'inondation et préserver la qualité des écosystèmes (Fletcher et al., 2013). Les noues, des larges fossés souvent végétalisés, font aujourd'hui partie des TA de plus en plus plébiscitées par les aménageurs, notamment pour leurs capacités de traitement des matières en suspension (MES) et des éléments traces métalliques (ETMs) (Fardel et al., 2019). Plusieurs types de noues se sont développés depuis une trentaine d'années, comme les noues de transport pour réguler les débits et les noues de filtration, optimisées pour l'épuration des eaux de ruissellement (Gavrić et al., 2019). Néanmoins, leurs conceptions restant assez empiriques, il convient d'étudier de manière approfondie leurs performances hydrologiques et épuratoires, notamment pour optimiser leur construction, leur implantation et leur maintenance. En ce sens, des travaux récents en France se sont intéressés (i) à l'influence du choix de la végétation (noue enherbée, noue plantée) sur les performances épuratoires des noues (Leroy et al., 2016) et (ii) aux performances épuratoires d'une noue de biofiltration vis-à-vis de la pollution particulaire et dissoute (Flanagan et al., 2018).

Pour d'une part améliorer les connaissances sur les fonctionnements hydraulique et épuratoire des noues et d'autre part évaluer l'impact de certains facteurs sur leurs performances, un pilote expérimental comprenant une noue standard et une noue de filtration a été aménagé au CSTB en décembre 2016. De tels dispositifs offrent non seulement l'avantage de maîtriser pleinement la quantité et la qualité des eaux injectées dans les ouvrages, mais ils facilitent aussi la mise en place d'une instrumentation adaptée aux objectifs de l'étude.

À cet égard, l'objectif de cet article est de présenter le fonctionnement de la chaîne de mesure qui a été mise en œuvre sur le pilote du CSTB. La reproductibilité du fonctionnement des deux noues est ensuite discutée à travers quelques résultats expérimentaux, acquis par 6 simulations d'un même évènement pluvieux. Ce travail pourrait être utile aux expérimentateurs puisqu'il initie une meilleure compréhension des sources de variations affectant une réponse obtenue sur tout ouvrage du même type.

\section{Materiels et méthodes}

\subsection{Dispositif pilote}

Deux noues ont été aménagées fin 2016 au sein de box étanches $(7 \times 4 \times 1,6 \mathrm{~m})$ situés sur la plateforme d'essai du CSTB, Aquasim. La méthode de construction de la première noue (notée REF pour «référence») vise à reproduire le terrassement d'un terrain naturel, donnant une noue «standard» capable de réguler le transport du ruissellement vers l'aval (Revitt et al., 2017). Cette dernière est constituée de sol excavé sur site, caractérisé comme un loam limoneux dans la classification texturale internationale (FAO, 2006). La seconde noue est assimilée à une noue de filtration (notée FILT); elle comprend des talus en loam limoneux ainsi qu'un massif central constitué d'une couche de filtration (sable lavé $0-2 \mathrm{~mm}$ ) recouvrant une couche drainante (gravier lavé 10-20 mm), selon les préconisations délivrées par le Facility for Advancing Water Biofiltration (FAWB, 2009). Dans les deux noues, la stabilité de chaque strate de matériau est assurée aux interfaces par des géogrilles (mailles de $500 \mu \mathrm{m}$ ). Les deux noues sont longues de $7 \mathrm{~m}$, présentent une section trapézoïdale, sont dotées d'un fond large de $1 \mathrm{~m}$ incliné de $1,6 \%$ et de talus de pente $25 \%$ (largeur de $80 \mathrm{~cm}$ ) (Fig. 1). L'épaisseur des couches de sol varie, après tassement naturel du massif, de 1,2 m (centre de la noue) à 1,4 m (bords de la noue). Pour suivre l'ensemble des flux d'eau et de polluants rejetés en aval des ouvrages, chaque noue a été équipée (i) d'un caniveau d'évacuation des eaux de surface et (ii) de huit drains annelés (diamètre $80 \mathrm{~mm}$ ) positionnés en fond d'ouvrage, au sein d'une couche de $10 \mathrm{~cm}$ de graviers. Tous les rejets du pilote sont évacués vers une galerie de mesure instrumentée. 
Tableau 1. Caractéristiques de l'instrumentation du pilote et détails sur la procédure de post-traitement.

\begin{tabular}{|c|c|c|c|}
\hline Paramètres & Technologie & $\Delta P^{\text {a }}$ & Post-traitement \\
\hline Pluie & Pluviomètres & $10 \mathrm{~min}$ et $30 \mathrm{~s}$ & voir Flori (2016) \\
\hline Débit entrée $\mathrm{REF}\left(\mathrm{Q}_{\mathrm{e} 3}\right)$ & DEM (4-20 mA) & $10 \mathrm{~s}$ & $\begin{array}{l}\mathrm{Q}_{\mathrm{e} 3}=\mathrm{Q}_{\mathrm{e} 1}{ }^{\mathrm{b}}-\mathrm{Q}_{\mathrm{e} 2} \\
\mathrm{Q} \text { moyenné sur } 1 \text { et } 5 \mathrm{~min}\end{array}$ \\
\hline Débit entrée FILT $\left(\mathrm{Q}_{\mathrm{e} 2}\right)$ & DEM (4-20 mA) & $10 \mathrm{~s}$ & $\mathrm{Q}_{\mathrm{e} 2}$ moyenné sur 1 et $5 \mathrm{~min}$ \\
\hline Débits de fuite & DEM (4-20 mA) & $10 \mathrm{~s}$ & $\mathrm{~A}_{x}{ }^{\mathrm{c}} \leq 0,028 \mathrm{~L} / \mathrm{s}: \mathrm{Q}_{\mathrm{f}}=\mathrm{Q}_{\text {augets }}$ \\
\hline REF et FILT $\left(Q_{f}\right)$ & Augets (contact sec) & & $\mathrm{A}_{x}{ }^{\mathrm{c}}>0,028 \mathrm{~L} / \mathrm{s}: \mathrm{Q}_{\mathrm{f}}=\mathrm{Q}_{\mathrm{DEM}}{ }^{\mathrm{d}}$ \\
\hline Volume entrée REF $\left(\mathrm{V}_{\mathrm{e} 3}\right)$ & $\operatorname{DEM}(0-5 \mathrm{~V})$ & $10 \mathrm{~s}$ & $\mathrm{~V}_{\mathrm{e} 3}=\mathrm{V}_{\mathrm{e} 1}{ }^{\mathrm{e}}-\mathrm{V}_{\mathrm{e} 2}$ \\
\hline Volume entrée FILT $\left(\mathrm{V}_{\mathrm{e} 2}\right)$ & DEM $(0-5 \mathrm{~V})$ & $10 \mathrm{~s}$ & $\mathrm{~V}_{\mathrm{e} 2}$ \\
\hline Volume sorties REF et FILT & $\operatorname{DEM}(0-5 \mathrm{~V})$ & $10 \mathrm{~s}$ & $\mathrm{~A}_{x}{ }^{\mathrm{c}} \leq 0,28 \mathrm{~L} / 10 \mathrm{~s}: \mathrm{V}_{\mathrm{f}}=\mathrm{V}_{\text {augets }}{ }^{\mathrm{f}}$ \\
\hline$\left(\mathrm{V}_{\text {fdrain }}, \mathrm{V}_{\text {fsurface }}\right)$ & Augets (contact sec) & & $\begin{array}{l}\mathrm{A}_{x}{ }^{\mathrm{c}}>0,28 \mathrm{~L} / 10 \mathrm{~s}: \mathrm{V}_{\mathrm{f}}=\mathrm{V}_{\text {DEM }}{ }^{\mathrm{g}} \\
\mathrm{V}_{\text {fdrain }}=\mathrm{V}_{\text {augets }}+\mathrm{V}_{\text {DEM }} \\
\mathrm{V}_{\text {fsurface }}=\mathrm{V}_{\text {augets }}+\mathrm{V}_{\text {DEM }}\end{array}$ \\
\hline Teneur en eau volumique $\theta_{\mathrm{v}}$ & Mesure de la permittivité diélectrique & $1 \mathrm{~min}$ & Formule constructeur (polynôme de degré 1 ) \\
\hline $\begin{array}{l}\text { Volume de déclenchement de } \\
\text { la collecte }\left(\mathrm{V}_{\mathrm{p}}\right)\end{array}$ & Asservissement au volume de passage $\left(\mathrm{V}_{\mathrm{i}}\right)$ & En direct & $\begin{array}{l}\mathrm{Q}_{\text {DEM }}{ }^{\mathrm{d}} \leq 0,026 \mathrm{~L} / \mathrm{s}: \mathrm{V}_{\mathrm{i}}=\mathrm{V}_{\text {augets }}{ }^{\mathrm{f}} \\
\mathrm{Q}_{\text {DEM }}{ }^{\mathrm{d}}>0,026 \mathrm{~L} / \mathrm{s}: \mathrm{V}_{\mathrm{i}}=\mathrm{V}_{\text {DEM }}{ }^{\mathrm{g}}\end{array}$ \\
\hline
\end{tabular}

${ }^{\text {a }}$ Pas de temps d'acquisition;

b Débit mesuré par un DEM en sortie de cuve :

c Débit mesuré par un système à augets basculeurs ;

${ }^{\mathrm{d}}$ Débit mesuré par un DEM;

e Volume d'eau mesuré par le DEM en sortie de cuve;

${ }^{\mathrm{f}}$ Volume d'eau mesuré par un système à augets basculeurs ;

g Volume d'eau mesuré par un DEM.

\subsection{Instrumentation du pilote}

\subsubsection{Suivi des volumes, des débits et de la pluviométrie}

En entrée de pilote, l'acquisition des paramètres hydrauliques (volumes et débits) est opérée par deux débitmètres électromagnétiques (DEM, diamètre nominal (DN) $50 \mathrm{~mm}$, Watermaster, $\mathrm{ABB}$ ) (Fig. 1). En sortie, un système couplant DEM (DN $25 \mathrm{~mm}$, FEW, ABB) et augets basculeurs (modèle 3039, Précis Mécanique) a été installé pour mesurer une gamme de débits variant de 0 à $0,7 \mathrm{~L} / \mathrm{s}$ (sortie de surface) ou de 0 à $0,3 \mathrm{~L} / \mathrm{s}$ (sortie de drains) (Fig. 1). D'après leurs constructeurs, les DEM DN 25, DEM DN 50 et les augets basculeurs présentent des plages de mesure respectives de 0,013 à $5,5 \mathrm{~L} / \mathrm{s}, 0,044$ à $21,9 \mathrm{~L} / \mathrm{s}$ et 0 à $0,028 \mathrm{~L} / \mathrm{s}$. Le choix du niveau de précision des DEM, $5 \%$, correspond à l'incertitude maximale garantie par ABB sur les plages de mesure. Par ailleurs, la calibration de chaque système à augets (volume d'un auget $=20 \mathrm{~mL}$ ) a été réalisée à plusieurs reprises (novembre 2017, janvier 2018 et mars 2018) en versant un volume de $500 \mathrm{~mL}$ d'eau. Le bon fonctionnement des augets a été validé lorsque le nombre de basculements se situe dans l'intervalle $[24 ; 26]$, assurant une précision de mesure de l'appareil de l'ordre de $5 \%$.

Sur le pilote, les données hydrauliques sont collectées par une centrale d'acquisition CR3000 (Campbell Scientific) toutes les 10 secondes. Les mesures sont ensuite post-traitées par un algorithme spécialement conçu sur MATLAB pour moyenner les débits et sélectionner les mesures provenant des DEM et des augets, en fonction de la gamme de débit considérée (Tab. 1). Par ailleurs, les ouvrages étant exposés aux précipitations atmosphériques, la pluviométrie locale est relevée via deux pluviomètres installés à proximité du pilote (Flori, 2016). Les données pluviométriques sont utilisées pour caractériser en partie l'état initial des deux noues (via le volume d'eau précipité dans les $24 \mathrm{~h}$ précédant un essai) et intégrer les précipitations dans les entrées des bilans hydrologiques.

\subsubsection{Suivi de l'état hydrique du sol}

L'état hydrique du sol est suivi en continu grâce à l'installation de sondes capacitives de teneur en eau volumique (EC-5, Decagon, volume de mesure $=0,2 \mathrm{~L}$ ). Chaque noue comprend 22 sondes réparties pour l'essentiel (20 EC-5) suivant des profils verticaux préalablement définis. Chaque profil est constitué de 4 sondes EC-5 introduites à $45^{\circ}$ dans le sol à $10,25,50$ et $75 \mathrm{~cm}$ de profondeur. Un des talus accueille 2 EC-5 supplémentaires enfouies à $25 \mathrm{~cm}$ de profondeur. L'acquisition des données s'effectue toutes les minutes et les données sont ensuite post-traitées sur MATLAB (Tab. 1). Les mesures des EC-5 sont utilisées pour estimer l'état de saturation effective $(\mathrm{Se})$ du sol, calculé avec la formule de Van Genuchten (1980):

$$
\mathrm{S}_{\mathrm{e}}(\%)=100 \times \frac{\theta_{\mathrm{v}}-\theta_{\mathrm{r}}}{\phi-\theta_{\mathrm{r}}}
$$

avec $\phi$ la porosité du sol $\left(0,44 \mathrm{~m}^{3} \cdot \mathrm{m}^{-3}\right.$ pour le sable; $0,33 \mathrm{~m}^{3} \cdot \mathrm{m}^{-3}$ pour la terre excavée), $\theta_{\mathrm{v}}$ la teneur en eau volumique $\left(\mathrm{en} \mathrm{m}^{3} \cdot \mathrm{m}^{-3}\right)$ et $\theta_{\mathrm{r}}$ la teneur en eau résiduelle $\left(0,02 \mathrm{~m}^{3} \cdot \mathrm{m}^{-3}\right.$ pour le sable ; $0,015 \mathrm{~m}^{3} \cdot \mathrm{m}^{-3}$ pour un loam limoneux d'après Rawls et al., 1982).

\subsubsection{Collecte des évènements}

Tous les échantillons collectés sur le pilote sont de type composite dans le but d'analyser des concentrations moyennes 
évènementielles (notée EMC). Contrairement aux eaux d'alimentation, collectées manuellement toutes les $12 \mathrm{~min}$ dans la cuve, les eaux de sortie sont prélevées automatiquement au sein d'un bac de collecte. Le fond du bac est relié à une tête d'échantillonneur (modèle ISCO 3710) via un tuyau en Téflon, permettant d'asservir les prélèvements à des volumes de passage. Son déclenchement entraîne le pompage d'un volume de $500 \mathrm{~mL}$ d'eau dans le bac, par la suite équitablement réparti dans deux flacons de $10 \mathrm{~L}$ (un en verre et un en plastique) pour analyser les contaminants inorganiques et organiques.

\subsection{Simulateur de ruissellement}

Le pilote a été équipé d'un dispositif spécifiquement conçu pour simuler des évènements de ruissellement. Il comprend un réseau en PVC équipé de plusieurs jeux de vannes, connecté en amont à une cuve d'alimentation d'environ $7 \mathrm{~m}^{3}$. Un bassin de rétention d'eau de toiture (toiture en acier galvanisé de $2500 \mathrm{~m}^{2}$ ) de $200 \mathrm{~m}^{3}$, bâché, est utilisé pour le remplissage de la cuve. Lors d'un essai, les eaux de la cuve sont injectées dans le réseau d'alimentation par une pompe de relevage. Deux pompes vide-cave sont utilisées en continu pour homogénéiser la qualité de l'eau dans la cuve. Le réseau en PVC dessert deux rampes d'injection pensées pour reproduire au choix un ruissellement de sortie de buse (alimentation en tête) ou de bord de route (alimentation latérale).

\subsection{Evaluation de la fonctionnalité de I'instrumentation et de la reproductibilité des performances}

La fonctionnalité de l'instrumentation a été évaluée en simulant à 6 reprises le même évènement de ruissellement, produit par une pluie d'intensité constante de $2 \mathrm{~h}$ et d'intensité $5,4 \mathrm{~mm} / \mathrm{h}$ (i.e. pluie de période de retour $<1$ an à Nantes). Rapportée à une surface contributive fictive de $200 \mathrm{~m}^{2}$, imperméabilisée à $90 \%$, un tel évènement génèrerait $1944 \mathrm{~L}$ d'eau en entrée de noue d'après la méthode rationnelle. Deux tests successifs sont séparés par un délai minimal de 22 h. Les 6 essais se déroulent préférentiellement par temps sec. Il est cependant toléré qu'une petite pluie (volume d'eau généré $\leq 5 \%$ du volume injecté) intervienne pendant les deux heures d'alimentation. Tout volume d'eau de pluie pendant et après un test est pris en compte dans les bilans volumiques. Les essais de performance épuratoire (caractérisation des flux de polluants en entrée/sortie de noue) ont été majoritairement effectués par temps sec ( $2 / 3$ des essais) pour limiter les apports en polluants des retombées atmosphériques, non quantifiés dans cette étude. En outre, pour réduire l'influence d'un évènement antérieur, le démarrage d'un essai a été astreint à un critère de nondépassement de $5 \%$ du débit maximal attendu par sortie au cours de l'essai.

Les réponses hydrauliques des six évènements sont caractérisées au travers de bilans volumiques et de suivis de l'évolution de Se à 10 et $75 \mathrm{~cm}$ de profondeur. Les capacités épuratoires des noues sont caractérisées au travers d'abattements en concentration (RE_c) et en masse (RE_m) :

$$
\mathrm{RE} \_\mathrm{c}(\%)=100 \times\left(1-\frac{\mathrm{C}_{\text {out }}}{\mathrm{C}_{\mathrm{in}}}\right) \text {, }
$$

$$
\mathrm{RE} \_\mathrm{m}(\%)=100 \times\left(1-\frac{\mathrm{M}_{\mathrm{out}}}{\mathrm{M}_{\mathrm{in}}}\right),
$$

avec

$$
\mathrm{M}_{\mathrm{in}}=\mathrm{C}_{\mathrm{in}} \times \mathrm{V}_{\mathrm{in}}
$$

et

$$
\mathrm{M}_{\text {out }}=\mathrm{C}_{\text {out }} \times \mathrm{V}_{\text {out }},
$$

avec $C_{\text {in }}$ et $C_{\text {out }}$ les concentrations totales en zinc ( $\mathrm{Zn}$ ) ou en cuivre $(\mathrm{Cu})$ d'entrée et de sortie mesurées par spectrométrie d'émission optique (ICP-OES) et/ou spectrométrie de masse (ICP-MS), $\mathrm{M}_{\text {in }}$ et $\mathrm{M}_{\text {out }}$ les masses d'entrée et de sortie, et $\mathrm{V}_{\text {in }}$ et $\mathrm{V}_{\text {out }}$ les volumes d'eau impactés par les prélèvements des échantillons composites.

Les abattements RE_c et RE_m ont été calculés sur les trois derniers essais pour $\mathrm{Cu}$ et $\mathrm{Zn}$. Les concentrations inférieures aux limites de quantification (LQ) sont estimées égales à la moitié des LQ pour procéder aux bilans de masse (Passeport et al., 2013).

Une première approche de l'analyse de la reproductibilité d'un résultat repose sur l'évaluation de la dispersion de ses valeurs «brutes ». Elle s'appuie sur un test de normalité, le test de Shapiro-Wilk. Si la valeur- $p$ obtenue est supérieure à 0,05 , la limite de reproductibilité $\mathrm{R}$, représentant l'écart maximal acceptable entre deux résultats, est estimée à partir de l'écarttype de la distribution ( $\sigma$ ) (Arnal, 2017):

$$
\mathrm{R}=2,83 \times \sigma
$$

Pour évaluer l'impact des précipitations atmosphériques dans la reproductibilité des réponses hydrauliques, les évolutions du ratio volume infiltré en 24 h/volume ruisselé (I), du ratio volumique entre les flux sortant et injecté artificiellement (S/E) et des différents Se ont été comparées à celles de deux paramètres liés aux précipitations atmosphériques: le ratio volume d'eau précipité pendant les $24 \mathrm{~h}$ suivant le départ du test/volume d'eau simulé (Cp) et le ratio volume d'eau précipité dans les $24 \mathrm{~h}$ précédant le début de l'essai/volume d'eau simulé (Ap24). Les intervalles de confiance à $95 \%$ (IC $95 \%$ ), estimés à partir des incertitudes de mesures des paramètres des indicateurs $\mathrm{S} / \mathrm{E}$, I et $\mathrm{Se}$ (Tab. 2), sont par la suite intégrés à l'évaluation de la reproductibilité. Ils facilitent la visualisation des marges d'erreur liées aux équipements de mesure et peuvent expliquer les écarts de résultats obtenus entre deux essais indépendants.

Des variations de la réponse hydraulique d'une noue pouvant affecter la représentativité d'une collecte d'échantillons composites, les efficacités des prélèvements ont été déterminées avec l'équation (7) pour évaluer l'impact de ces variations sur les abattements massiques.

$$
\text { Efficacité prélèvement }(\%)=100 \times \frac{\mathrm{V}_{\text {out }}}{\mathrm{Vt}}
$$

avec Vt le volume total rejeté (sortie de surface de la noue standard) ou le volume atteint lorsque la différence horaire du 
Tableau 2. Modélisation des incertitudes sur les variables mesurées sur le pilote ou sur les indicateurs de performance des noues pilotes.

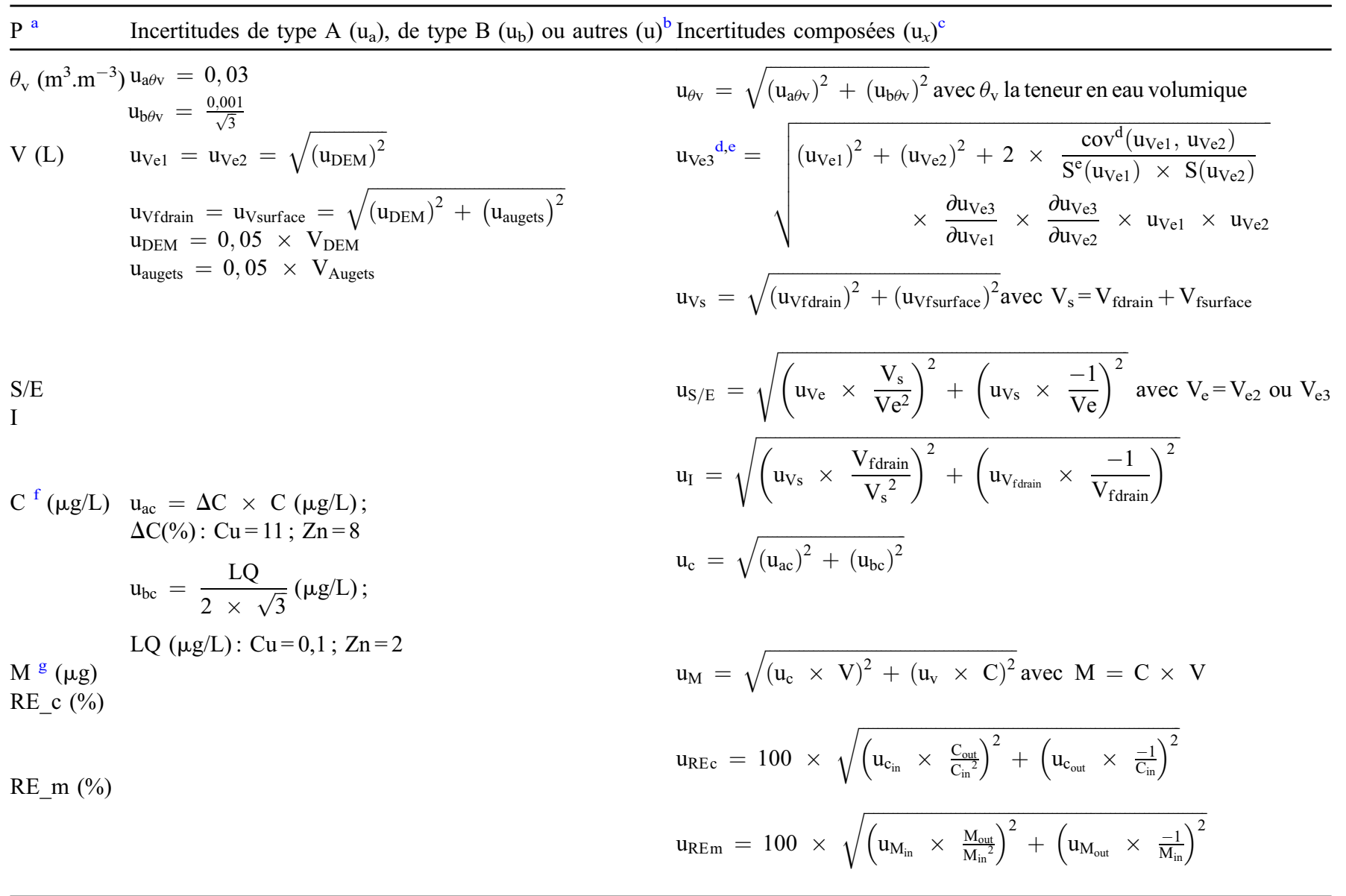

a Paramètre;

b Incertitudes calculées suivant les procédures du JCGM (2008);

c Utilisation de la méthode de propagation des incertitudes (JCGM, 2008), l'incertitude composée obtenue est ensuite multipliée par 2 pour estimer les IC $95 \%$;

${ }^{\mathrm{d}}$ Covariance des deux variables calculées sur les séries de données construites à partir des six essais (uniquement pour la noue standard);

e Écart type calculé sur les séries de données construites à partir des six essais ;

${ }^{\mathrm{f}}$ Concentration du contaminant dans l'eau;

g Masse du contaminant injectée ou rejetée.

volume drainé par la noue est inférieure à $1 \%$ du volume total drainé.

En outre, les marges d'erreur des valeurs d'abattement, liées à la quantification des flux de polluants, ont été estimées via leurs IC $95 \%$.

\section{Resultats et discussion}

\subsection{Fonctionnement de la chaîne de mesure hydraulique lors d'un essai}

La relative stabilité des débits d'entrée autour de $0,27 \mathrm{~L} / \mathrm{s}$ (Fig. 2) valide la capacité du dispositif à alimenter simultanément les deux noues avec un débit constant. Seul le début de l'essai présente une non-conformité du débit avec la valeur objectif, nécessitant de la part de l'opérateur d'ajuster le diamètre d'ouverture des vannes positionnées en entrée de pilote. Les variations de débit observées suite à ce réglage sont inhérentes à celles de la vitesse de l'eau dans la canalisation, probablement liées à des imperfections de fonctionnement de la pompe de relevage pendant l'essai.

Le système de mesure des flux d'eau en sortie, composé d'un DEM et d'un système à augets, s'avère apte à restituer la réponse hydraulique des ouvrages. Le couplage de deux appareils de mesure, dotés de plages de fonctionnement complémentaires (faibles débits pour les augets et débits modérés à forts pour le DEM), a permis de caractériser les trois périodes de la réponse hydraulique d'une noue: (1) une montée en débit couverte par le système à augets puis le débitmètre électromagnétique, (2) un palier hydraulique mesuré par le débitmètre électromagnétique et (3) un drainage de l'ouvrage, majoritairement restitué par les augets (Fig. 2). Cette couverture intégrale des réponses hydrauliques facilite le choix des volumes de déclenchement des préleveurs. Ces derniers ont pu être programmés de sorte à ce que leurs plages de collecte recouvrent les différentes 


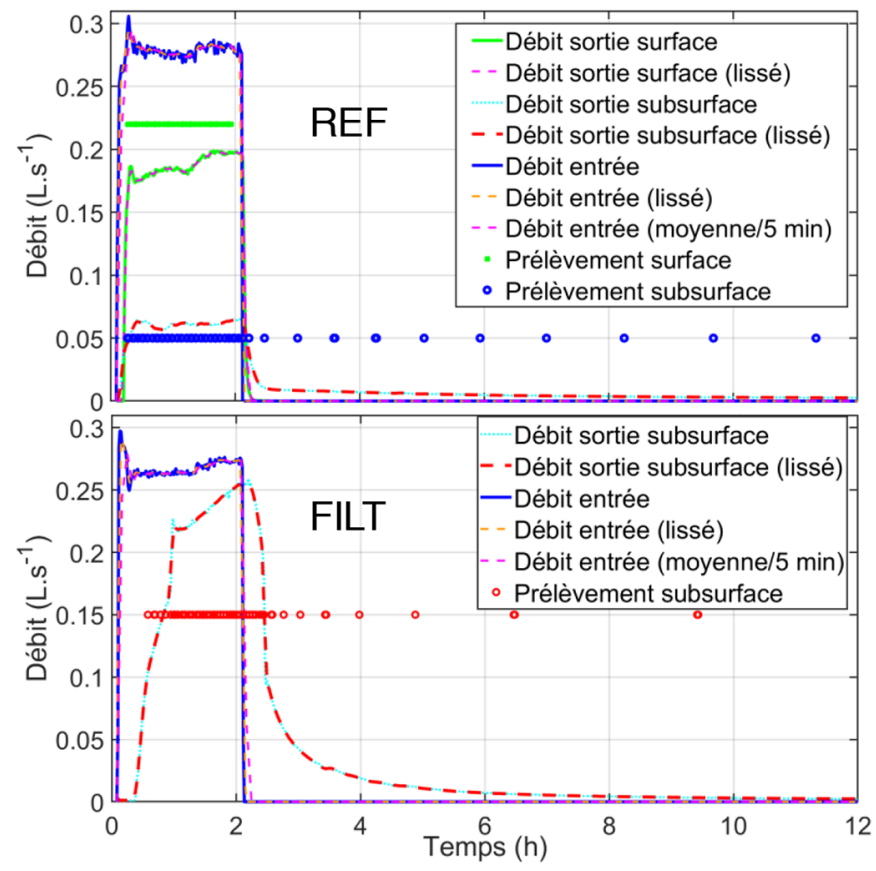

Fig. 2. Réponses hydrauliques des noues standard REF et de filtration FILT; les préleveurs sont déclenchés tous les $29 \mathrm{~L}$ (surface REF), $17 \mathrm{~L}$ (subsurface REF) et $47 \mathrm{~L}$ (subsurface FILT).

phases de la réponse hydraulique (Fig. 2). Par conséquent, le système de mesure des débits rend possible la caractérisation fine des flux de contaminants apportés par un évènement au sein des ouvrages.

\subsection{Evaluation de la reproductibilité du fonctionnement hydraulique}

Les données acquises par le pilote peuvent être utilisées pour caractériser le fonctionnement hydraulique des noues. Les données brutes de l'indicateur $\mathrm{S} / \mathrm{E}$, proches de 1 pour la noue standard (moyenne $=1,00$ ) et pour la noue de filtration (moyenne $=1,01$ ), indiquent que les deux ouvrages sont capables de drainer en moins de $24 \mathrm{~h}$ l'évènement de ruissellement simulé (période de retour $<1$ an) (Fig. 3). Les résultats du test de Shapiro-Wilk n'ont pas permis de rejeter l'hypothèse de non normalité des distributions de $\mathrm{S} / \mathrm{E}$ pour les deux noues. La faible dispersion des $\mathrm{S} / \mathrm{E}$ (coefficient de variation $(\mathrm{cv})<4 \%$ ) suggère une possible bonne reproductibilité de ce ratio entre deux essais indépendants. Cette hypothèse est confirmée pour la noue standard, aucun écart entre les $\mathrm{S} / \mathrm{E}$ issus de deux essais indépendants ne dépassant la limite de reproductibilite $\left(\mathrm{R}_{\mathrm{S} / \mathrm{E}}\right)$. Pour la noue de filtration, un dépassement du $\mathrm{R}_{\mathrm{S} / \mathrm{E}}$ est atteint entre les évènements du 26/03 et du 28/03 (Fig. 3). Il coïncide avec un état de saturation initial de la noue plus élevé pour l'essai du 28/03, probablement en raison de la pluie ayant généré $331 \mathrm{~L}$ avant son départ. Une partie de cet apport naturel d'eau au massif s'est probablement additionnée au flux drainé provenant de l'évènement simulé, augmentant automatiquement $\mathrm{S}$ et donc $\mathrm{S} / \mathrm{E}$. Par conséquent, une pluie précédant un essai est susceptible d'entraver la reproductibilité du bilan hydraulique d'une noue. Des conditions de départ d'essai plus strictes (p.ex. Ap24=0) ou une couverture des ouvrages pour les protéger de la pluie pourraient remédier à ces variations.

En outre, les marges d'erreur de $\mathrm{S} / \mathrm{E}$ dues aux équipements de mesure des flux volumiques sont à considérer pour expliquer en partie les variations des S/E. En effet, les étendues des IC $95 \%$ sont significatives, représentant jusqu'à $30 \%$ (noue standard) et $15 \%$ (noue de filtration) de la valeur du critère $\mathrm{S} / \mathrm{E}$. L'incertitude 2 fois plus grande de $\mathrm{S} / \mathrm{E}$ pour la noue standard s'explique par la mesure indirecte (différence des données provenant de deux DEM) de ses flux d'entrée et par des flux de sortie mesurés en deux points de la noue (sorties du caniveau et des drains). Ce dernier élément confirme, à conditions initiales similaires, le rôle de la chaîne d'instrumentation de mesure dans les variations potentielles du bilan hydraulique. Il serait donc souhaitable de limiter autant que possible le nombre d'appareils de mesure et l'acquisition de paramètres par mesures indirectes.

Du point de vue du fonctionnement hydraulique des deux noues, la distribution entre infiltration et ruissellement présente une faible dispersion $(\mathrm{cv}<5 \%)$. Néanmoins, la part d'infiltration la plus élevée a été enregistrée lors de la présence d'une pluie dans les $22 \mathrm{~h}$ suivant l'essai ( $\mathrm{I}=39,4 \%$ le 27/11/2012). Cet essai a induit un dépassement de la limite de reproductibilité de $\mathrm{I}\left(\mathrm{R}_{\mathrm{I}}\right)$ (Fig. 3). L'apport naturel d'eau par une pluie est donc susceptible d'entraver la reproductibilité du ratio I. En revanche, la prise en compte des IC $95 \%$ ne permet pas de détecter de variations significatives de ce ratio. Il est à noter que les IC $95 \%$ de I sont plus faibles que ceux de S/E $(\approx 10 \%)$, en raison du mode direct d'acquisition des données, qui proviennent uniquement de deux systèmes de mesure.

Concernant les évolutions des Se au cours d'un essai, les variations entre les trois temps considérés $(0,2$ et $24 \mathrm{~h})$ sont plus visibles pour la noue de filtration, du fait de $\mathrm{S}_{\mathrm{e}}$ initiaux souvent inférieurs à $30 \%$. Les pics de saturation du massif, observés en fin d'alimentation ( $\mathrm{Se} 2 \mathrm{~h}$ ), sont plus élevés sur les campagnes de mars/avril pour la noue de filtration. Cette différence s'expliquerait par la modification de l'état de surface de l'ouvrage (retrait de tensiomètres initialement présents), assurant une meilleure répartition du flux d'eau au sein du massif sableux. Néanmoins, les valeurs élevées des IC $95 \%$ $( \pm 20 \%)$ et le faible volume de mesure d'une sonde EC-5 $(0,2 \mathrm{~L})$ nuisent à la détection plus fine des variations temporelles de l'état de saturation des noues (Fig. 3). Pour y remédier, il pourrait être envisagé de remplacer les EC-5 par des capteurs à plus haut volume d'intégration.

\subsection{Evaluation de la reproductibilité d'indicateurs de performances épuratoires}

La dispersion des indicateurs de performances épuratoires est globalement meilleure pour $\mathrm{Zn}(\mathrm{cv}<30 \%)$ que pour $\mathrm{Cu}$ $(-185 \% \leq \mathrm{cv} \leq 176 \%)$ (Fig. 4). L'hypothèse de normalité de l'ensemble des distributions d'abattements n'a pas pu être rejetée par la méthode de Shapiro-Wilk (valeur- $p>0,05$ ). Aucun dépassement des limites de reproductibilité n'a été observé entre deux abattements issus d'évènements indépendants. Pour $\mathrm{Cu}$, la plus forte variabilité observée sur les abattements serait principalement due aux faibles concentrations analysées en entrée (environ 2,5 $\mu \mathrm{g} / \mathrm{L}$ ) et en sortie $(2,6$ 

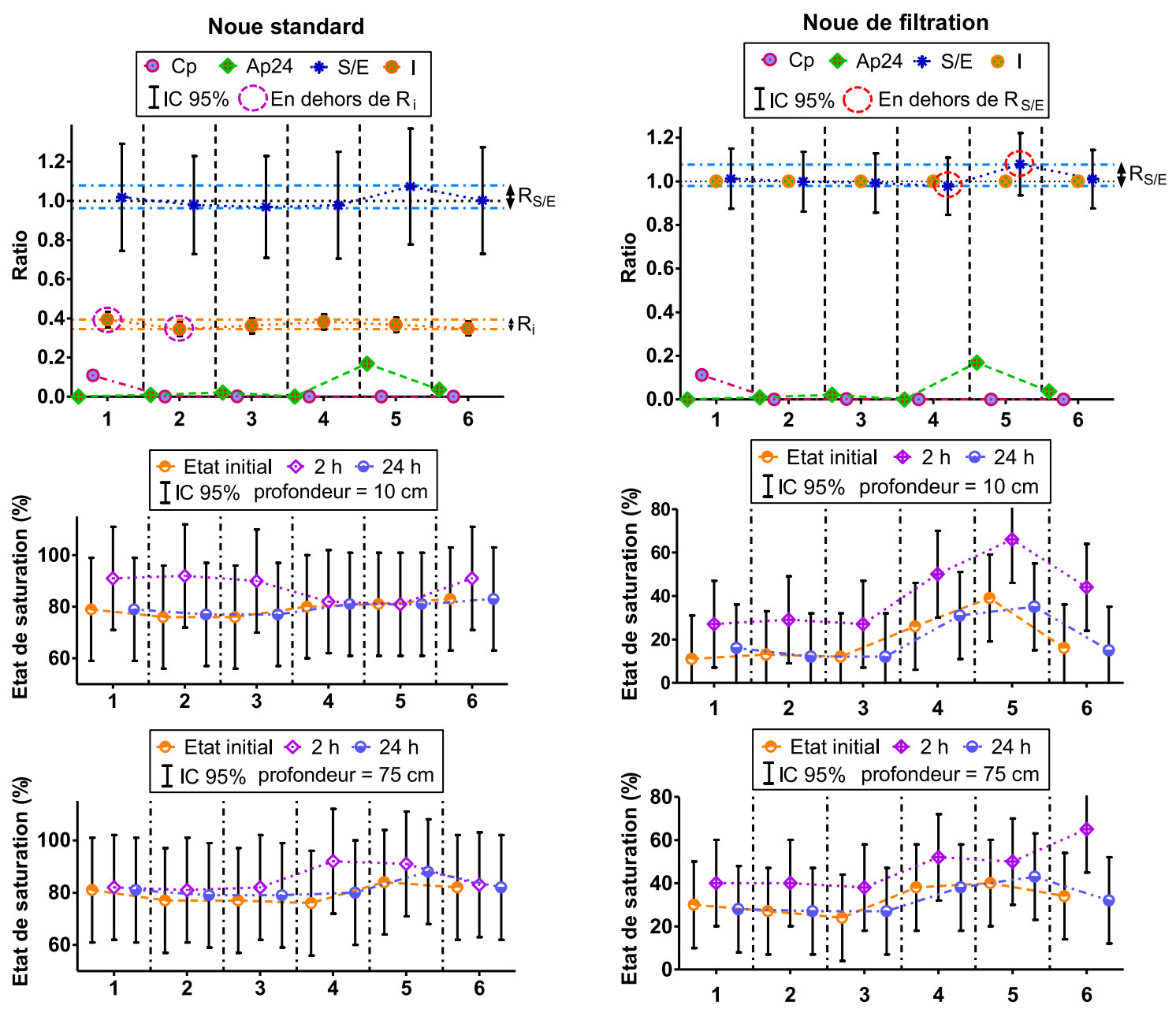

Fig. 3. Evaluation de la reproductibilité du fonctionnement hydraulique des deux noues lors des six essais simulés $(5,4 \mathrm{~mm} / \mathrm{h}$ pendant $2 \mathrm{~h})$. Les numéros en abscisse indiquent des dates de tests sur le pilote $(1: 27 / 11 / 2017,2: 29 / 11 / 2017,3: 01 / 12 / 2017,4: 26 / 03 / 2018,5: 28 / 03 / 2018,6$ : 05/04/2018).

à $6,5 \mu \mathrm{g} / \mathrm{L})$ de noues. Les fluctuations des efficacités de prélèvement (comprises entre 87 et $102 \%$ ) semblent très peu influer sur celles des abattements massiques. Par ailleurs, les incertitudes liées aux suivis des flux d'eau et à la quantification des concentrations en $\mathrm{Zn}$ et en $\mathrm{Cu}$ doivent être considérées pour expliquer les variations de performances. En les prenant en considération, les performances sont alors dans l'ensemble reproductibles. Il est à noter que les IC $95 \%$ sont d'autant plus forts que les concentrations en polluants sont faibles en entrée de noue ou que les abattements sont faibles. Ce résultat rappelle que les meilleures performances sont souvent caractérisées avec des niveaux de contamination des eaux élevés (Fardel et al., 2019).

\section{Conclusions}

Cette étude a permis d'évaluer la reproductibilité des réponses hydrauliques et épuratoires de deux noues pilotes soumises à 6 reprises au même évènement de ruissellement. Lors de chaque essai, les systèmes de mesure des débits
(DEM + augets basculeurs) ont permis de restituer intégralement la réponse hydraulique de chaque noue, améliorant la plage d'asservissement des échantillonneurs utilisés pour la caractérisation des flux de polluants. Les bilans et le fonctionnement hydraulique des deux noues sont reproductibles dans 3 à 5 des 6 essais en fonction des paramètres considérés. La reproductibilité des ratios hydrauliques $\mathrm{S} / \mathrm{E}$ et I a pu être affectée par une pluie significative (volume $>10 \%$ du volume d'alimentation) précipitée sur la noue avant ou après l'essai. L'essai impacté par une pluie avant son départ fut aussi marqué par une teneur en eau à saturation initiale plus élevée. Par conséquent, les précipitations atmosphériques précédant un test ainsi que l'état hydrique initial des matériaux pourraient, dans certains cas (évènements pluvieux extrêmes, sol proche de la capacité au champ ou de la saturation), entraver significativement la reproductibilité de la réponse hydraulique. Compte tenu de la faible dispersion de la teneur en eau initiale dans cette étude, des essais complémentaires pourraient être mis en place pour avoir une image plus complète de son influence sur le fonctionnement hydraulique d'une noue. Par ailleurs, la modification de l'état de surface de 

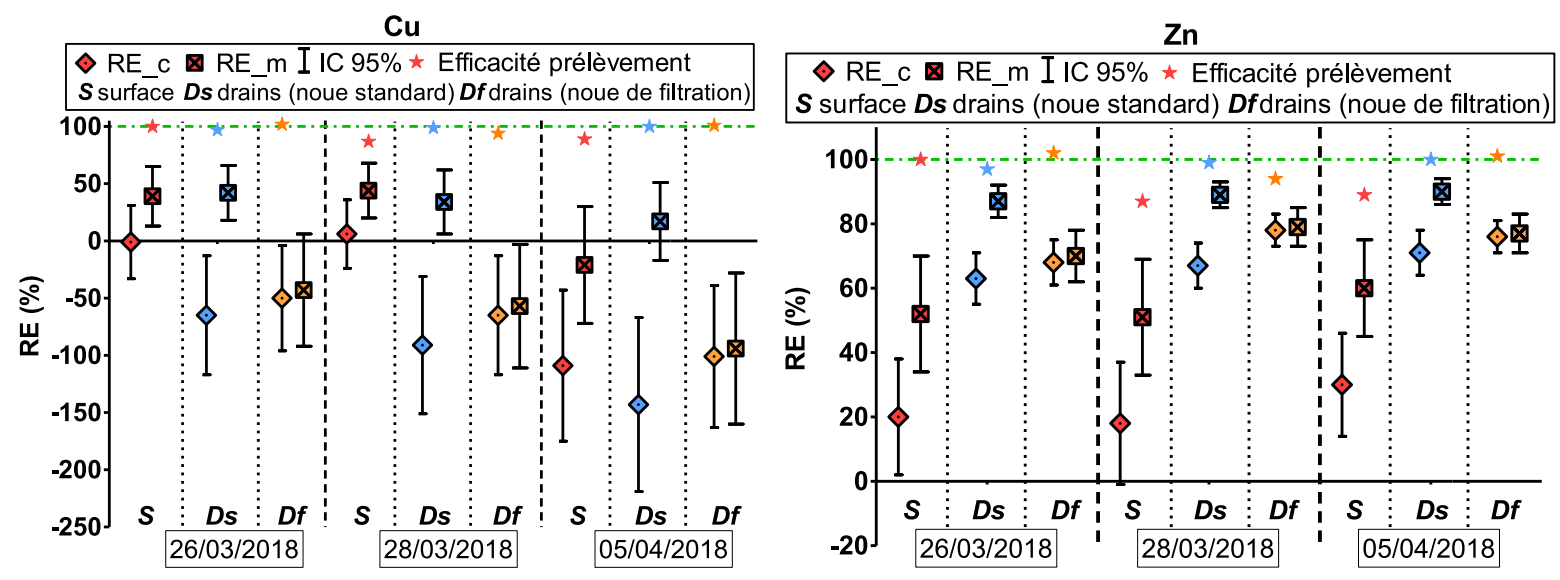

Fig. 4. Evaluation de la reproductibilité des indicateurs de performances épuratoires pour $\mathrm{Cu}$ et $\mathrm{Zn}$.

la noue de filtration en cours d'étude a entraîné une différence de répartition du flux d'eau en son sein. De plus, les niveaux élevés de certaines incertitudes liées à la chaîne de mesure du pilote (p.ex. jusqu'à $30 \%$ du ratio $\mathrm{S} / \mathrm{E}$ ) seraient aussi à considérer pour affiner l'évaluation de la reproductibilité des paramètres calculés.

Concernant les facteurs interférant sur la reproductibilité des performances épuratoires liées à ces essais, les incertitudes dues aux méthodes d'analyse et à la chaîne de mesure des flux d'eau contribueraient pour partie aux variations d'abattements observées. En outre, le niveau de contamination des eaux en entrée de noue pourrait influer sur la dispersion des résultats de performances épuratoires. Cette hypothèse reposant uniquement sur le comportement de deux polluants $(\mathrm{Cu}$ et $\mathrm{Zn})$, il serait intéressant d'enrichir l'analyse en faisant varier la charge polluante en entrée de pilote et en suivant d'autres types de contaminants (nutriments, pesticides, micropolluants émergents).

Remerciements. Cette étude a été conduite dans le cadre du projet Matriochkas, financé par l'Agence Française pour la Biodiversité. Les auteurs remercient vivement le personnel technique du LEE et du CSTB, notamment Frédéric Padiou pour son expertise technique lors de la conception et l'aménagement du banc d'essai.

\section{Références}

Arnal F. 2017. Répétabilité \& reproductibilité d'une méthode. Disponible sur http/flarnal.e-monsite.com/medias/files/repet-1.pdf.

FAO. 2006. Guidelines for soil description. Rome: Food and Agriculture Organization of the United Nations.

Fardel A, Peyneau PE, Béchet B, Lakel A, Rodriguez F. 2019. Analysis of swale factors implicated in pollutant removal efficiency using a swale database. Environ Sci Pollut Res 26(2): 1287-1302.
FAWB. 2009. Guidelines for soil filter media in biofiltration systems (Version 3.01). Melbourne, Australia: Facility for Advancing Water Biofiltration, Monash University.

Flanagan K, Branchu P, Boudahmane L, Caupos E, Demare D, Deshayes S, et al. 2018. Field performance of two biofiltration systems treating micropollutants from road runoff. Water Res 145: 562-578.

Fletcher TD, Andrieu H, Hamel P. 2013. Understanding, management and modelling of urban hydrology and its consequences for receiving waters: A state of the art. Adv Water Resour 51: 261-279.

Flori JP. 2016. Approche temporelle fine des statistiques de précipitation. La Houille Blanche 1: 65-76.

Gavrić S, Leonhardt G, Marsalek J, Viklander M. 2019. Processes improving urban stormwater quality in grass swales and filter strips: A review of research findings. Sci Total Environ 669:431-447.

JCGM. 2008. Evaluation of measurement data-Guide to the expression of uncertainty in measurement, (First edition). Joint Committee for Guides in Metrology (Working Group 1), JCGM 100:2008. Disponible sur https://www.bipm.org/utils/common/ documents/jcgm/JCGM_100_2008_E.pdf.

Leroy MC, Portet-Koltalo F, Legras M, Lederf F, Moncond'huy V, Polaert I, et al. 2016. Performance of vegetated swales for improving road runoff quality in a moderate traffic urban area. $S c i$ Total Environ 566-567: 113-121.

Passeport E, Tournebize J, Chaumont C, Guenne A, Coquet Y. 2013. Pesticide contamination interception strategy and removal efficiency in forest buffer and artificial wetland in a tile-drained agricultural watershed. Chemosphere 91: 1289-1296.

Rawls WJ, Brakensiek DL, Saxtonn KE. 1982. Estimation of soil water properties. Trans ASAE 25: 1316-1320.

Revitt DM, Ellis JB, Lundy L. 2017. Assessing the impact of swales on receiving water quality. Urban Water $J$ 14: 839-845.

Van Genuchten MT. 1980. A closed-form equation for predicting the hydraulic conductivity of unsaturated soils. Soil Sci Soc Am J 44: 892-898.

Citation de l'article : Fardel A, Peyneau P-E, Béchet B, Lakel A, Rodriguez F. 2019. Evaluation de la reproductibilité des réponses hydraulique et épuratoire de deux noues pilotes. La Houille Blanche 2: 27-34. 